\title{
Analysis on the Color Components of Rural Landscape Based on Visual Perception
}

\author{
Hongli Zhu, ${ }^{1, *}$ \\ ${ }^{1}$ Yunnan Institute of Economics and Management, Kunming, Yunnan 650106, China \\ *Corresponding author. Email: zhuhongli8811@163.com
}

\begin{abstract}
This article analyzes the color components of the rural landscape from the perspective of visual perception objectively. Through the psychological empathy effect of color perception and visual psychological stimulation, the visual perception effect of color is analyzed, and the components of the rural landscape color are explored. It starts with the source of the rural landscape, the combination of the color elements of the rural landscape, the composition of the natural background color elements of the rural landscape, and the composition of the artificial background color elements of the rural landscape. It needs to re-understand the regional natural and human characteristics of the rural landscape, and focuses on the constituent elements of the rural landscape, which is the rural landscape. The color components provide the basis for the color gamut.
\end{abstract}

Keywords: visual perception, rural landscape, color composition, natural background color, artificial

background color

\section{INTRODUCTION}

At present, more and more rural landscape construction methods tend to be similar, and many rural landscape cultures with local characteristics are missing, which brings great challenges to the development and inheritance of Chinese rural civilization to a certain extent. As the carrier of the region and spirit, it has accumulated a profound cultural heritage from life and has distinct regional characteristics. Therefore, the interpretation of the color components of the rural landscape can make the design work more distinctive.

This paper focuses on the analysis and sorting of the color components of the rural landscape based on the perception of visual perception, discusses the principles and construction methods of the color components of the rural landscape. It can enrich and supplement the theoretical system of rural landscape design. Through the in-depth analysis of the color components of the rural landscape research and summarizing the research results, we hope to have reference significance for the design of the color components of the rural landscape.

\section{THE VISUAL PERCEPTION EFFECT OF COLOR}

A. The psychological empathy effect of color
Vicher, Smasov, etc. believe that when people observe external things through their visual organs, they often have "resonance" or "community" in their hearts. The appropriate emotion is the psychological empathy effect of aesthetics, or the theory of "empathy effect" in aesthetics. Studies have shown that the empathy effect of color on the environment is obvious. Some scientists have found that color can affect brain waves. Brain waves are alert to red and relax to blue, and people can perceive cold and perceive the warmth of the sun and fire is a kind of psychological feeling and reaction of people to natural colors.

Therefore, rational use of the psychological empathy effect of color on visual perception constitutes the color components of the rural landscape. By using the principles of individualized color, color matching, color contrast, etc., the design principles of the rural landscape are constructed and the appropriate color that arouses emotional resonance is found. The rules of collocation, rational use of the combination, difference, contrast, emphasis and other interactions between colors form a stable unity of colors. Based on the empathy effect of these colors, they are reasonably used in the elements of the rural landscape, and some common colors are mastered. The characteristics of empathy and psychological perception, better use the characteristics of color language to shape the rural landscape environment.

*Fund project: Scientific research fund project in Yunnan province department of education, Project No: 2019J0964. 


\section{B. Visual mental stimulation perception of color}

Color of stimulating the role and influence of the environment on the human body, includes external sensory stimulation, sensory stimulation within a comprehensive internal and external sensory stimulus (i.e., mental stimulation); color stimulus is from the outside of the course of conduct of environmental stimuli cause. Different color tones have different stimuli to people. When the stimulus is moderate, people will actively adjust themselves. When the stimulus is too large, people will actively adjust or change the environment, or even create new ones.

In the rural landscape color composition, the general large-scale landscape background didn't use bright colors. The most designed have soft neutral color tone, or used to create a harmonious atmosphere and comfortable environment. There are rural landscapes based on visual perception, psychology and aesthetic principles in the design. Mainly consider the coordination of the main color and the secondary color, highlight the main color, and use the secondary color as a foil to form an aesthetic effect with obvious color contrast and coordinated color matching.

\section{Inherent superiority of color vision psychology}

Different colors to different views of people experiencing some color in different contexts, will have inherent advantages. The dark colors can more easily access people's attention than bright colors, just like the strange novel shape more easily attract attention than bright colors. There are similar things, for example, the familiar styling with bright material objects can be more attractive than rough objects, and rhythmic color combinations can make people more comfortable than haphazard color combinations. Colors have infiltrated the complex thoughts, emotions and life experience of human beings, and become rich in culture and connotation. Corresponding emotional associations will naturally arise. For example, green not only gives people a sense of youth and vitality, but also gives people peaceful, calm and fresh, youthful, long grass, healthy and energetic, positive cultural association. Whether it is the physiological feeling of color or cultural association, it affects people's behavioral response and evaluation.

Therefore, design should be good at choosing the right color from the perspective of cultural psychology in the local landscape. Based on this characteristic, local people have a good mental impression of the design landscape works. According to the requirements of different experiences of the landscape, it' necessary to adjust the color composition element or background of the theme, and choose the appropriate color design to reflect the attributes and regional characteristics of the village itself.

\section{THE COLOR COMPONENTS OF THE RURAL LANDSCAPE}

\section{A. The source of vernacular landscape colors}

Color comes mainly from the rural landscape of plants, plant leaves and is a native landscape design in the largest color area for the longest time. Therefore, it's decided to plant the native landscape of the overall color tone. The plant landscape of flowers and ornamental fruit has seasonality that can not only enrich the color and enjoyment of the landscape, but also highlight the characteristics of the rural landscape in the color tone. Besides, the bark color of deciduous trees will also become the most important color composition in the landscape.

Rural landscapes include rocks, water, landscape sketches, paving color, etc. The proportion of rural landscapes is small. They have more types of color and more complex changes to adapt to the local landscape. The contrast in degree is both coordinated and prominent. Natural water is colorless, but affected by the area, depth, water quality, sunshine and environment, the water body will show different colors. Artificial water bodies can also be laid with different pool bottom materials and lighting as needed to create different artistic concepts. Landscape sketches are mostly composed of agricultural landscape-related objects or sculptures, which mainly enrich the characteristics and affinity of rural landscapes.

\section{B. Rural landscape color combination of elements}

The general color combinations of the rural landscape and landscape design space, design conception, and artistic expression are closely linked. The same or similar color expresses tonal unity, harmony, simple, contrast weak. The different shades of the color combination are similar to reconcile, just like spring green leaves, or golden autumn leaves. By the shadows in the light or jungle shrubs in a backlight with coordination, contrasting color can combine and emphasize color area, depth and distribution relationships. Color concentration can enhance the effect and can play a decorative color dispersion. To reconcile the role, the most important principle is the balance of color combinations. Different color combinations can result in local different styles of the landscape.

Rural landscape color elements combined with rural village field and color complementary field color is the plane on the ground constituted, is a combination of color, texture and shape, and the ecological field is the main constituent elements of local landscape, but also the local landscape color combinations of elements important content, and it is not for the pure design and composition to do this, the color of disorder, asymmetry, uneven right had a harmonious beauty. 


\section{Composition of base color elements of rural landscape}

The base color of the rural landscape is mainly composed of the inherent colors of natural material entities such as mountains and waters, woods, plants, buildings, fields, rivers, and landscape water. The sky is the background of the landscape environment and a flowing picture. The color changes are affected by clouds and fog. The influence of time is greater. The natural land and fields are the most different "land" of the rural landscape environment space, reflecting the characteristics of different spatial environments. Together, the land and the sky will accompany the people living in the natural environment from beginning to end, and affect the rural environment. The landscape effect is also a decisive basic color system in the rural landscape, so it has become an indispensable part of the entire rural environment. At the same time, the mountains and waters, woods, plants, buildings, fields, rivers, and landscape water that exist in the land and the sky are the most poetic and high-profile colors in the rural environment. They are all living entities with life, and will change with their growth stages. change their color change season. It is the best choice to create dynamic color countryside village landscape. It can be the starting point of color and broken color effect.

\section{Local landscape area human color elements constitution}

In the rural settlements and the environment, the natural landscape color of the rural landscape exists as a regional and background color. Therefore, the color characteristics of the regional humanistic landscape must have the subordination under the macro natural background and the micro regional conditions.

In the environment of vernacular buildings and structures, and in a relatively small area, buildings, structures, clothing, and signs have become the background of the activities of the vernacular landscape. In the construction of specific landscapes, architecture is the topical part of the landscape environment. It is the most conspicuous attraction, so the architectural color is correspondingly the protagonist of several landscape colors, and its color processing directly affects the beauty of the scenic colors. For buildings with different functions, the color choices are also different, and we must strive to achieve the perfect combination of color and function. In addition, the choice of architectural colors should make full use of the characteristics of commonality, contrast, sequence, primary and secondary colors, so that the architectural colors are full of changes and distinctive features, thereby enhancing the attractiveness of the rural environment.
Farming tools or life of equipment is a local landscape composed of human color elements important part of the elements. The farming utensils or life of equipment as the leading traditional agricultural way of life has disappeared, and farming utensils or appliances life is socially eliminated because of loss of function. However, as a tangible "symbol" of farming civilization, farming tools have the dual meaning and value of history and culture, and they also play an important role in the humanistic color of rural landscapes.

\section{CONCLUSION}

In short, we will deeply study the individual differences of local regional rural landscapes, pay attention to its inherent original ecological color system, extract the most dazzling color characteristics, and evolve and enlarge it through the artificial landscape in the village, combining ecological techniques, ecological materials, folk customs, etc. Natural colors, maximize the use of original ecological ground materials, highlight the natural color of the wood stone of the architectural color, such as light yellow, cyan gray, gray blue, etc. Appropriately increasing the sky blue, bright yellow, red and other medium-brightness colors of some rural landscapes, is to activate the overall color of the rural landscape. It can effectively meet the people of rural landscape diversity adventures psychology. Using the visual perception theory, we can summarize the main problems of color-used in the local landscape, and analyze the combined color characteristics and sensory evaluation. We can sum up the principle of optimization of color landscape, and hope to make some innovative and breakthrough research about the topic, and hope to achieve sustainable and healthy development of the rural landscape.

\section{References}

[1] Raymond et al: "Studies on children's room design psychological effects of color vision," [J], "design", 2013 Year 6, pp. 150 .

[2] Zhu Yue, Zhang Huarong, Lansi Jen, Huangqi Tang. based on quantitative analysis and public perception of the traditional village color landscape Optimization - A Case Study of Taiwanese crab Po Tsuen [J], Central China Normal University ( Natural Science Edition ). 2020, 01 : 50-59.

[3] Dong Shuge, Li xiao. Study the development of circular economy industrial chain to promote the construction of rural ecological civilization $[\mathrm{J}]$. Learn Chinese Society for Environmental Sciences surgery Proceedings Annual Meeting, 2014, 7: 592-595

[4] Zhu Hongli. Talking about the application of visual psychology in residential interior design [J]. Interior Design, 2007, 3.

[5] Dong Lijing. City "open landscape" of visual perception research [J]. Green Technology 2020, 15: 22-25 pages. 
[6] Wang Yili. Visual perception of graphic design expression evaluation method based on $[\mathrm{J}]$. Changchun Institute of Technology (Natural Science) 2020, 02: 125-128 pages.

[7] Liu Bo. The control of color elements in the Changxiang soil landscape: Taking Pingbaying Forest Park as an example [J]. Forum of Science and Technology Association (second half of the month). 2010, 01: 116-117. 